\title{
Impacto da Seleção de Funções de Bases Radiais em Formulações de Elementos de Contorno aplicadas a Modelos Advectivo-Difusivos
}

\author{
Pinheiro, V. P. ${ }^{1,3 *}$;Loeffler, C.F. ${ }^{1}$; Neves, N.S. ${ }^{2}$ Almeida, L.M. ${ }^{4}$ \\ 1 PPGEM/UFES -Programa de Pós-Graduação em Engenharia Mecânica, Universidade Federal do \\ Espírito Santo, Vitória, ES, Brasil. \\ 2 COPPE/UFRJ - Instituto Alberto Luiz Coimbra de Pós-Graduação e Pesquisa de Engenharia, \\ Programa de Engenharia Civil, Universidade Federal do Rio de Janeiro, RJ, Brasil. \\ 3 CCTM/IFES - Coordenadoria do Curso Técnico em Mecânica, Instituto Federal do Espírito Santo, \\ Campus Guarapari, ES, Brasil. \\ 4 Programa de Iniciação Científica em Métodos Numéricos, Universidade Vila Velha, Vila Velha, ES, \\ Brasil.
}

* e-mail: vitor.pinheiro1987@gmail.com

\section{Resumo}

As formulações do Método de Elementos de Contorno (BEM) baseadas em aproximações via funções de bases radiais tais como a clássica técnica de Dupla Reciprocidade (MECDR) e a mais recente formulação de Interpolação Direta (MECID) tem ganhado cada vez mais campo de aplicação na engenharia e meio científico, devido a sua flexibilidade e robustez frente a modelos matemáticos diversos. Neste contexto a investigação da eficiência de certas funções de bases radiais na abordagem de um problema físico em específico, por meio destas formulações, é de interesse contínuo. Neste artigo pretende-se investigar de maneira inicial a performance das funções radial simples $r$, radial cúbica $r^{3}$ e radial de placa fina $r^{2} \ln r$, em problemas advectivosdifusivos, com campo de velocidade constante, e que utilizem as formulações MECDR e MECID. A precisão de cada formulação com o uso das distintas bases radiais supracitadas é avaliada em relação a soluções analíticas bem conhecidas da literatura.

Palavras-Chave: Interpolação Direta, Funções de Bases Radiais, Elementos de Contorno, Advecção-Difusão.

\section{Introdução}

A abordagem de modelos advectivosdifuvisos pelo Método de Elementos de Contorno (MEC) experimentou muitos avanços científicos nas últimas duas décadas. Em meados do anos oitenta, uma formulação mais clássica do método, que utiliza uma solução fundamental correlata ao problema físico [1] mostrouse capaz de representar a dominância da advecção, sem instabilidades numéricas. A desvantagem desta abordagem era a limitação da solução fundamental utilizada à problemas de velocidade constante.
Nos anos noventa, as formulações com aproximações de integrais de domínio via bases radiais, como a Dupla Reciprocidade (MECDR), proposta por Nardini e Brebbia [2], conferiram versatilidade e amplitude ao MEC [3], sobretudo com a possibilidade de descrever campos hidrodinâmicos variáveis, mas ainda limitadas número de Peclet mais reduzidos.

Paralelamente, as funções de bases radiais (FBR) foram ganhando contorno no cenário científico, incialmente como uma ferramenta de interpolação de um conjunto de dados espalhados [4], e com grande desenvolvimento nos anos subse- 


\section{Blucher Proceedings XI Encontro Científico de Física Aplicada

quentes, o que permitiu a expansão de sua aplicabilidade para diversas vertentes, tal como incentivou o surgimento de diversas novas funções, tanto de suporte global, tal como compacto [5],[6]. Com isso a aplicação dessas funções no Método de Elementos de Contorno se tornou cada vez mais difusa.

Mais recentemente, uma nova formulação baseada em aproximações por bases radiais, proposta por Loeffler [10], denominada Interpolação Direta (MECID) mostra-se capaz de prover bons níveis gerais de precisão numa ampla gama de problemas de campo escalar [7].

No contexto de modelos de avecçãodifusão, significativos avanços foram trazidos pela MECID, em especial no quesito estabilidade numérica frente a efeitos intensificados de convecção, com boa performance em escoamentos com moderados números de Peclet [8].

Neste artigo pretende-se testar a performances FBR clássicas da classe Spline e Thin Plate Spline [9] em um problema advectivo-difusivo de velocidade constante, sob as formulações MECDR e MECID. O objetivo é bipartido entre avaliar a precisão das bases radiais e a resposta das mesmas em situações de efeitos advectivos mais significativos.

\section{Interpolação Direta}

Nesta seção aborda-se a formulação integral da técnica MECID, uma vez que a MECDR já é bem difusa no meio científico [3]. A modelagem matemática é inerente a um problema advectivodifusivo em meio homogêneo e com campo de velocidade uniforme.

$$
\lambda u,_{i i}=v_{i} u,_{i} \quad \lambda=\frac{k}{\rho c_{p}}
$$

Na equação $1, \lambda$ representa a difusividade térmica do meio, $k$ a condutividade térmica, $\rho$ a massa específica, $c_{p}$ o calor específico a pressão constante e $v_{i} \mathrm{o}$ campo hidrodinâmico de velocidade.

A multiplicação da equação 1 pela por uma solução auxiliar $u^{*}$ e posterior integração ao longo do domínio gera a formulação integral forte do problema, na equação 2.

$$
\int_{\Omega} u,{ }_{i i} u^{*} d \Omega=\int_{\Omega} v_{i} u,{ }_{i} u^{*} d \Omega
$$

O lado esquerdo da equação 2 contém os efeitos difusivos e seu tratamento integral inverso é amplamente relatado em literatura. [11]. Na MECID, tal como na MECDR, $u^{*}$ é a solução do problema fundamental de Laplace [12].

$$
c_{\xi} u_{\xi}+\int_{\Gamma} u q^{*} d \Gamma-\int_{\Gamma} q u^{*} d \Gamma
$$

O lado advectivo é submetido a um processo de regularização [13], mostrado a seguir pela equação 4 , que promove um tratamento da singularidade quando o ponto campo $u$ coincide com o ponto fonte $u \xi$.

$$
\begin{array}{r}
\int_{\Gamma} v_{i} n_{i} u u^{*} d \Gamma-\int_{\Omega} v_{i} u,{ }_{i}^{*} u d \Omega \\
+\int_{\Omega} v_{i} u,{ }_{i}^{*} u \xi d \Omega-\int_{\Omega} v_{i} u,{ }_{i}^{*} u \xi d \Omega
\end{array}
$$

O formato final da equação 4 , que quantifica a parte advectiva, após as devidas manipulações pós-regularização, e condensadas as integrais é:

$$
\begin{array}{r}
\int_{\Gamma} v_{i} n_{i} u u^{*} d \Gamma-\int_{\Omega} v_{i} u,,_{i}^{*}\left[u-u_{\xi}\right] d \Omega \\
-\int_{\Omega} v_{i} u,{ }_{i}^{*} u \xi d \Omega
\end{array}
$$

A filosofia da Interpolação Direta propõe uma aproximação de todo o núcleo da integral de domínio de interesse. Na equação 5 , o foco é aproximar a segunda integral regularizada. A aproximação da MECID tem o formato da equação 6 , e a escolha da função de base radial é uma linha de pesquisa contínua, que inclusive motiva este trabalho. [14]. 


$$
v_{i} u,{ }_{i}^{*}[u-u \xi] \cong \alpha_{j}^{\xi} F_{j}\left(X_{j}, X\right)
$$

Os elementos de contorno utilizados para discretização são lineares isoparamétricos e as integrações numéricas realizadas com quadratura de GaussLegendre com 20 ponto de aproximação.

\section{Funções de Base Radial de Suporte Global}

Há um número elevado de tipos de formatos de funções de bases radiais para aplicações nos mais diversos ramos da física, matemática e engenharias. Estas funções podem ser agrupadas através de parâmetros, como por exemplo o tipo de suporte: global ou compacto [14]. O conceito de suporte diz respeito a extensão de interação de cada ponto com a nuvem de dados de forma geral, onde suporte global preconiza uma interação de cada elemento com todos os outros, paralelamente ao suporte compacto, onde esta interação é limitada a uma região específica [9].

As principais representantes das funções de base radial com suporte global são trazidas pela Tabela 1 , onde $n$ deve ser ímpar para a classe Spline Simples e par no caso de Spline de Placa Fina.

Tabela 1 - FBR's com Suporte Global

\begin{tabular}{|l|c|}
\hline \multicolumn{1}{|c|}{ FBR } & $F\left(X_{j} ; X\right)$ \\
\hline Spline Simples & $r^{n}$ \\
\hline Spline de Placa Fina & $r^{n} \ln r$ \\
\hline Multiquádrica & $\sqrt{1+r^{2}}$ \\
\hline Gaussiana & $e^{-r^{2}}$ \\
\hline
\end{tabular}

Como a técnica da Dupla Reciprocidade (MECDR) e da Interpolação Direta (MECID) utilizam as primitivas das FBR, um pequeno procedimento é necessário para deduzir estas funções auxiliares.

Igualando a FBR selecionada a um laplaciano em coordenadas polares, tal como na equação 7 , já considerando a simetria angular em $\theta$.

$$
\nabla^{2} \psi=\frac{\partial^{2} \psi}{\partial r^{2}}+\frac{1}{r} \frac{\partial \psi}{\partial r}=F\left(X^{j} ; X\right)
$$

$\mathrm{Na}$ sequência basta integrar sucessivamente, e com as considerações adequadas sobre as constantes, gerar a primitiva $\psi$ e sua derivada $\psi_{, i}$, que aparececem na formulações do MEC a serem testadas.

Neste trabalho as seguintes FBR de suporte global são testadas: radial simples $\mathrm{r}$, radial cúbica $r^{3}$ e radial de placa fina $r^{2} \ln r$. A seleção da FBR adequada a cada problema físico abordado constitui importante linha de pesquisa ativa. [15],[16]

\section{Estudo de Caso}

$\mathrm{O}$ experimento numérico é realizado na geometria quadrada mostrada abaixo, com dimensões unitárias e sujeita as condições contorno da Figura 1.

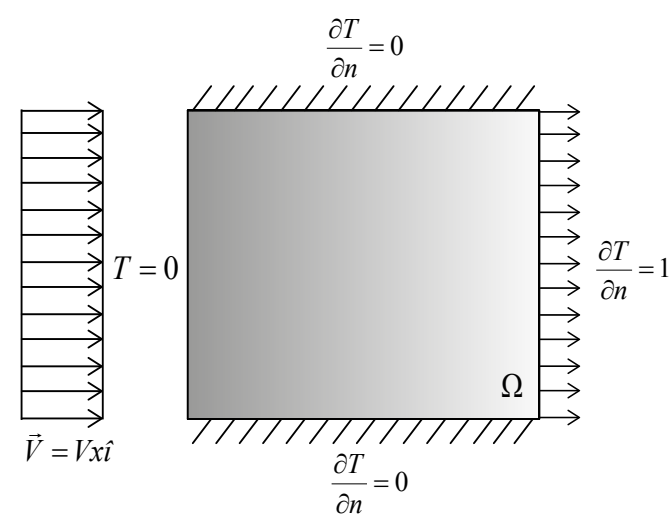

Figura 1: Domínio Computacional

Apesar do experimento em questão se tratar de um problema bidimensional em termos de geometria, o comportamento físico é unidimensional devido à simetria das condições de fluxo nulo aplicadas na arestas horizontais paralelas.

A priori, alguns pré-testes são executados a respeito da convergência, em relação aos dois parâmetros de malha: número de elementos de contorno (EC) e polos internos (PI). As sensibilidade das formulações MECDR e MECID à discretização do contorno é parelha, entretanto, o MECID é mais influenciado pela quantidade de pontos internos e requer uma maior quantidade dos mesmos. [7],[10],[13].

Com uma malha suficientemente refinada para uma boa performance das duas 


\section{Blucher Proceedings XI Encontro Científico de Física Aplicada

formulações, com 64 EC e 121 PI, inicia-se uma análise da precisão alcançada por cada uma das três FBR testadas em um contexto de gradual incremento dos efeitos da advecção. Inicia-se pela exposição dos resultados da técnica de Interpolação Direta, na Figura 2 a seguir.

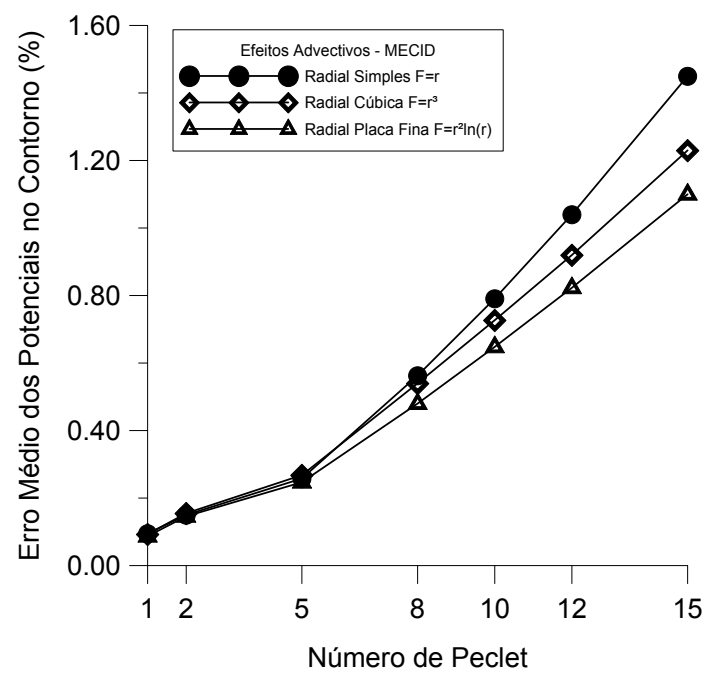

Figura 2: Formulação MECID

A Figura 2 revela que para a malha testada, de forma geral, os resultados são muito parecidos na MECID para as três FBR testadas, onde destaca-se a radial de placa fina (TPS), que apresenta melhor precisão, seguida da radial cúbica.

Já quanto a advecção, é interessante observar que o MECID, manteve níveis baixos de erros médios nos cálculos dos potenciais para todo o intervalo de números de Peclet testado, o que mostra uma boa estabilidade numérica frente à aceleração do escoamento.

Na sequência são exibidos pela Figura 3, os resultados para as três mesmas FBR com a formulação de Dupla Reciprocidade do MEC.

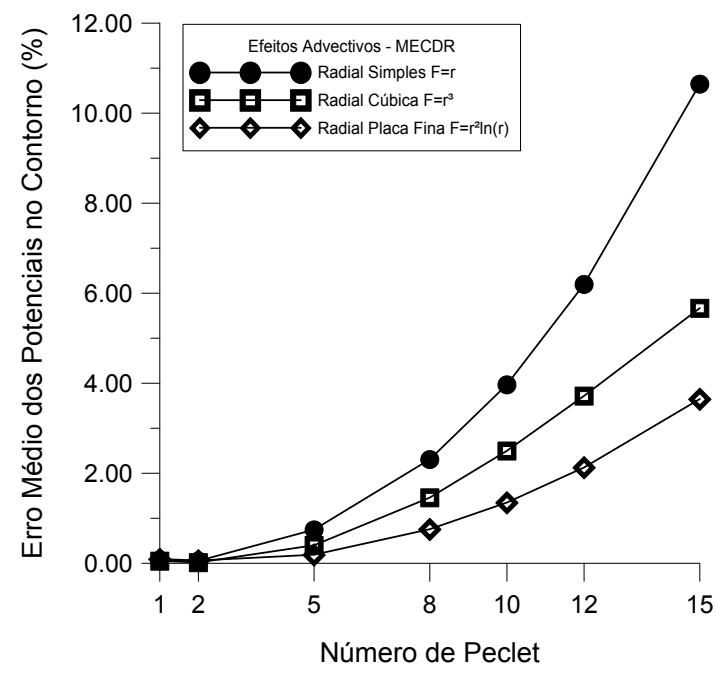

Figura 3: Formulação MECDR
Quanto as funções de base radial testadas, a MECDR mostrou-se muito mais sensível a seleção das mesmas. Isto pode ser observado pelas diferenças de erros médios entre as curvas, e explicado matematicamente pelas duas aproximações por bases radiais exigidas pelo modelo matemático da MECDR [3]. Repetese aqui a sequência de desempenho do teste com a MECID, onde a função de placa fina performa melhor, seguida pela radial cúbica.

Os níveis de erros médios gerados pela MECDR são superiores aos alcançados pela MECID nos mesmos cenários e crescem com uma velocidade muito superior. Tal constatação, alinhada com trabalhos anteriores [8], demonstra uma boa tolerância da formulação de Interpolação Direta a situações físicas que apresentem Peclet moderado, o que, por sua vez, constitui uma limitação clássica da técnica MECDR.

\section{Considerações Finais}

Os resultados preliminares mostrados nas análise deste artigo reforçam a importância da seleção de bases radiais na precisão nas formulações de elementos de contorno, tal como na MECID e MECDR. No caso testado, a MECDR mostrou-se mais influenciada pelo intercâmbio entre as bases radiais, relativamente ao MECID.

A formulação de Interpolação Direta (MECID) mostrou-se mais precisa e estável em relação à situações com efeitos advectivos mais severos, o que confere à formulação um potencial que ainda demanda testes mais sistêmicos. Experimentos numéricos com geometrias mais exigentes, meios nãohomogêneos, escoamentos compressíveis e não linearidades físicas são alguns dos novos desafios para a consolidação científica das características da MECID.

\section{Referências}

[1] Honma T., Tanaka Y., Kaji I. Regular boundary element solutions to steadystate convective diffusion equations. Engineering Analysis, 1985, vol.2, pp. 9599. 


\section{Blucher Proceedings XI Encontro Científico de Física Aplicada Blucher}

[2] Nardini D., Brebbia C.A. A new approach to free vibration analysis using boundary elements. Applied Mathematical Modelling, 1983, vol. 7, pp. 157162.

[3] Partridge P. W., Brebbia C.A., Wrobel L.C. The Dual Reciprocity, Boundary Element Method, Computational Mechanics Publications and Elsevier, London, UK, 1992.

[4] Hardy R.L. Multiquadric equations of topography and other irregular surfaces. J Geophys Res, 1971, Vol. 76, pp. 19051915.

[5] Hardy R.L. Theory and applications of the multiquadric-biharmonic method, 20 years of discovery, 1968-1988. Comp Math Applic, 1990, Vol. 19, pp. 163208.

[6] Wedland H. Piecewise polynomial, positive definite and compactly supported radial functions of minimal degree. Adv. in Comput. Math., 1995, Vol.4, pp. 389396.

[7] Loeffler, C. F., Barcelos, H. M., Mansur, W.J., Bulcão, A. Solving Helmholtz Problems with the Boundary Element Method Using Direct Radial Basis Function Interpolation. Engineering Analysis with Boundary Elements, 2015, Vol. 61, pp. 218-225.

[8] Pinheiro V.P. Application of the Boundary Element Method with Regularized Direct Integration to Two-dimensional Advective-Diffusive Problems. (in portuguese). Masters Thesis, 2018, Federal University of Espírito Santo.

[9] Biancolini M.E. Fast Radial Basis Functions for Engineering Applications.
Springer International Publishing AG, Italy , 2017.

[10] Loeffler, C. F., Cruz, A. L., Bulcão, A. Direct Use of Radial Basis Interpolation Functions for Modelling Source Terms with the Boundary Element Method. Engineering Analysis with Boundary Elements, 2015, vol. 50, pp. 97-108.

[11] Brebbia C. A., Telles J. C. F., Wrobel L.C. Boundary Element Techniques, First Ed., Springer-Verlag, Berlin Heidelberg, 1984.

[12] Kythe, O. J. An Introduction to Boundary Element Methods, CRC Press, Boca Ratton, USA,1995.

[13] Loeffler, C. F., Mansur, W. J. (2017). A regularization scheme applied to the direct interpolation boundary element technique with radial basis functions for solving eigenvalue problem. Engineering Analysis with Boundary Elements, 74, 14-18.

[14] Buhmann, M. D. Radial Basis Functions: Theory and Implementations, first ed., Cambridge University Press, New York, USA, 2003.

[15] Goldberg M.A., Chen C.S. The theory of radial basis functions applied to the BEM for inhomogeneous partial differential equations. BE Communication 5, 1994, pp. 57-61.

[16] Loeffler C.F., Zamprogno L., Mansur W. J., Bulcao A. Performance of Compact Radial Basis Functions in the Direct Interpolation Boundary Element Method for Solving Potential Problems. Computational Methods in Engineering Analysis, 2017, Vol. 133, pp. 367-387. 